\title{
Article
}

\section{What might Normalisation Process Theory bring to policy implementation studies? Learning lessons and uncovering questions through a case study of the profound implementation failure of a new policing policy}

Mackenzie, Mhairi, Bradley, Lisa, Stanley, Nicky, Gannon, Maria, Barton, Deborah, Cosgrove, Katie, Conway, Ellie and Feder, Gene Available at http://clok.uclan.ac.uk/24947/

Mackenzie, Mhairi, Bradley, Lisa, Stanley, Nicky ORCID: 0000-0002-7644-1625, Gannon, Maria, Barton, Deborah, Cosgrove, Katie, Conway, Ellie and Feder, Gene (2019) What might Normalisation Process Theory bring to policy implementation studies? Learning lessons and uncovering questions through a case study of the profound implementation failure of a new policing policy. Social Policy \& Administration, 53 (3). pp. 449-463. ISSN 0144-5596

It is advisable to refer to the publisher's version if you intend to cite from the work. http://dx.doi.org/10.1111/spol.12467

For more information about UCLan's research in this area go to and search for <name of research Group>.

For information about Research generally at UCLan please go to http://www.uclan.ac.uk/research/

All outputs in CLoK are protected by Intellectual Property Rights law, including Copyright law. Copyright, IPR and Moral Rights for the works on this site are retained by the individual authors and/or other copyright owners. Terms and conditions for use of this material are defined in the policies page. 
What might Normalisation Process Theory bring to policy implementation studies? Learning lessons and uncovering questions through a case study of the profound implementation failure of a new policing policy. 


\begin{abstract}
Normalisation Process Theory, used nationally and internationally to explore implementation within health services research, is used for the first time within policing to understand profound policy implementation failure and to generate broader discussion of policy implementation theory. The policy in question (Police To Primary Care) was an intervention designed to notify GPs when women are assessed by police as at high risk of future Domestic Abuse (DA). Designed to improve inter-agency communication, it took place amidst radical organisational change.
\end{abstract}

Using qualitative interviews with DA specialist and frontline officers, this paper addresses how NPT helps to explain the (non)implementation of P2PC; how such an analysis differs from other policy implementation approaches; and, what this means for our understandings of policy implementation more broadly.

NPT proved useful in understanding mechanisms leading to (non)implementation of the intervention: fuzzy alignment with existing practice; faulty communication of purpose; and, inattention to discretionary implementation spaces. It helped us understand why the intervention came to be invisible. Dwarfed by its organisational context, made institutionally hard-to-read by a lack of formal protocols, and given restricted view to police officers, it was compromised by a failure to instigate systems of organisational learning.

More broadly, NPT helped reveal practices intersecting top-down and bottom-up implementation theory. The paper concludes by asking how NPT and theories of street-level bureaucracy might be better used in tandem and, particularly, how this might help explorations of policy implementation where human actors are joined by technological actors in interpreting and making policy in vivo.

Key Words: Normalisation Process Theory, Domestic Abuse, Policy Implementation, Police, Technology, Organisational Change 


\section{Introduction}

This paper is concerned primarily with the role of Normalisation Process Theory (NPT) in understanding profound policy implementation failure, used for the first time in the context of policing, and in its potential contribution within broader debates of policy implementation. We start with the implementation problem which serves as the case for our deliberations.

In 2012 Strathclyde Police introduced a pilot whereby officers notified family doctors when women experienced domestic abuse (DA). The scheme, Police to Primary Care $(P 2 P C)$, required signed consent from women prior to the letter being sent. As conceived, P2PC sat comfortably within existing policy and practice; interagency referral was already routine for those attending DA incidents and was recognisable within a wider political appetite for DA policing to comprise a more joined-up, woman-centred approach. When it was introduced, however, there was radical organisational change to policing in Scotland culminating in the merger of eight territorial forces into Police Scotland, and smaller but significant changes to database systems and officers' administrative workloads. In 2013 we entered the field to evaluate the implementation of P2PC. In our earliest meetings with police staff, what became apparent was the profound failure of the scheme's introduction. Whilst early calculations suggested around 2,500 potential candidates for GP notification, only 92 cases were notified ${ }^{1}$. Later interviews would reveal that the majority of frontline staff had little knowledge of the scheme. Not simply had P2PC failed to deliver, it appeared to have burned-up on entry.

As researchers we were left with two dilemmas. Beyond stark failure, was there a policy implementation story worth telling? If so, what frame might best shape its narrative? We came to reconcile our expectations of policy implementation with the peculiar intervention that was P2PC and recognised it not simply as an example of policy implementation failure but one which expanded our understanding of policy (non)implementation. In response to the second dilemma, how we might frame the narrative, we utilised NPT (May and Finch, 2009) to discuss P2PC as an abandoned policy.

This is the first paper to apply NPT within the context of contemporary policing and to open up discussion concerning NPT's connections to other policy implementation models. In this paper we use it for two ends. First, NPT is deployed analytically to identify mechanisms 
which undermined the implementation of P2PC within Police Scotland, an organisation applauded for its leadership in tackling DA (Scottish Government, 2014). The second and more salient purpose of this article is to consider NPT's potential value for broader understandings of policy implementation.

We draw on data from P2PC's evaluation which addressed how the scheme was implemented and perceived by key stakeholder groups (author). This paper is concerned with a more methodological set of questions: does NPT help explain the (non)implementation of P2PC?; how does such analysis differ from other policy implementation approaches?; and, what might this mean for our understandings of policy implementation more broadly? We give a brief overview of policy implementation theory and how it relates to P2PC, before introducing NPT as our conceptual frame. We then analyse P2PC using NPT. This analysis then informs our discussion which considers what NPT helps make visible that might have remained absent; and, reflects on the value of NPT compared to other implementation lenses.

\section{The (non)implementation of policy: from familiar thinking to adopted frameworks}

\section{Understanding Implementation}

As Cairney (2012) argues, policy implementation studies are necessitated by the regular departure of practice from policy across all domains, including policing (Kirby, 2013). Theories of implementation 'gaps' have formed around two perspectives: top-down and bottom-up approaches. The top-down tradition takes policy to be that designed/defined in government spaces by elite officials; its associated aims are to examine the extent to which policy objectives have been achieved over time and, instrumentally, to improve policy implementation (Mazmanian and Sabatier, 1989). Bottom-up approaches contest the view that policies are fully formed entities made by high-level decision-makers, and instead retain focus on encounters between service-users and professionals working on the ground, viewing these the sites of policy emergence.

This latter tradition, typified by the study of street-level bureaucrats as per Lipsky (1980), is found in the work of more recent theorists, including Hupe and Hill (2015), Brodkin (2013). 
Their collective work provides a different angle from which to analyse the disjuncture between policy as conceived-on-paper and delivered-in-practice and questions the very rhetoric of the 'gap', making clear that practices of policy are just as important to policy making as the elite spaces in which they are devised (Rowe, 2012). Indeed, positioning the contribution of Street-level Bureaucracy in relation to an implementation 'gap' serves to reinforce a normative understanding of the policy process, whilst similarly, situating Lipsky's work within the top-down, bottom-up debate misses the more fundamental question that his book addresses: "how to understand complex organizational behaviour" (Brodkin, 2015, p. 941). Indeed, when policy is recognised as indeterminate, what becomes of interest is a much broader variety of factors that shape its implementation and its manifestation on delivery. Central to this work therefore are the concepts of discretion (the varied application of formal and informal rules by street-level bureaucrats), coping (the internal and external mechanisms street-level bureaucrats adopt in order to deal with conflicts and contradictions in proposed practices) and client-processing (the routines street-level bureaucrats adopt when categorising service-users) (Hupe and Hill, 2015).

There have also been long-standing efforts to synthesise these approaches. Sabatier, a selfconfessed "top-downer", sought to combine the two (1986), as did Elmore (1979). Others have opted for a mixed approach, designing criteria to determine whether top-down or bottom-up may be more appropriate. One example is Matland's ambiguity-conflict matrix (1995). He argues that disagreements between top-down and bottom-up approaches are essentially disagreements over what constitutes the correct object of research - "fidelity to the designer's plan or on the general consequences of the implementation actions?" ( $p$. 154). Concluding that "top-downers and bottom-uppers study different types of policies" ( $p$. 155), his response is a contingency model based on two factors: ambiguity and conflict. In brief, ambiguity attends to goals and means, whilst conflict considers actor agreement on goals. Policies can be categorised by researchers as one of four types across these two orthogonal axes.

Adding to these debates Gibb (2015) observes that, not surprisingly, understanding policy success has occupied more academic interest than conceptualising policy failure(s). Exceptions to this include the work of Gibb himself and that of King and Crewe (2013). Both 
success and failure, however, involve attributes related to King and Crewe's (2013)

distinction between structural factors (such as institutional weaknesses) and 'agency' factors (incorporating human 'error'). In this latter category ideas relating to discretion reside (although we note here our discomfort with the label of 'error' and with the binary separation of structure and agency). Nonetheless, definitions of what constitute failure, regardless of whether it is driven by macro, meso or micro level factors, may be contested depending on one's success criteria (McConnell, 2010) which might in turn have ideological or methodological origins - in the case of P2PC there is no room for debate; the policy in practice simply was not implemented to any meaningful degree.

\section{Implementation and P2PC}

More broadly, how might P2PC fit within these approaches and concepts? To give more background to the scheme, P2PC was jointly developed by Strathclyde Police (via its Domestic Abuse Coordination Unit (DACU))and NHS Health Scotland, aiming to arm GPs with knowledge to facilitate disclosure and improve health care. It was to sit within local Domestic Abuse Investigation Units (DAIU), with specialist DAIU officers visiting women deemed at high risk of future DA to obtain their signed consent for GP notification. At the earliest stages of P2PC's implementation, however, the lack of resources for DAIU officers to administer the scheme in this way became apparent, thus the task shifted mostly into the existing routines of frontline officers who were already gathering consent, albeit verbal, for other inter-agency referrals.

Prior to commencing the evaluation, our ontological sympathies rested with street-level theorists' concerns with frontline workers making not just delivering policy; we anticipated finding variations that would best be explained by discretionary practice in coping with changes to existing routines, and in processing individuals accordingly. Likewise, we found additional purchase in Matland's framework, and determining P2PC a low ambiguity/low conflict policy focused our attention on bottom-up considerations. There were certainly features of the context which encouraged such expectations, including the seeming simplicity of the intervention, its alignment with existing modes of working, and its relevance to broader social and political concerns. 
To elaborate: first, on paper, P2PC came across as manageable in size, had a short implementation-chain and appeared non-burdensome for officers. Because of the high number of DA cases in Scotland it had been agreed that, in the pilot phase, the scheme would apply to cases where a victim was considered at high risk of serious harm ${ }^{2}$ and we were told that a recognised police algorithm would determine such victims. Further, the information flow was unidirectional from the police to the relevant GP. GPs were not required to feedback their discussions to the police.

Second, such a model of police communication is common across the UK (for example, to social or advocacy services). As such, having specialist Domestic Abuse Investigation Unit (DAIU) officers (who already had responsibility for investigating crimes and supporting victims) responsible for GP referral as part of P2PC appeared perfectly in keeping with their role. Likewise, the practice of obtaining consent in order to send a notification letter to victims' GPs sat comfortably within existing practices.

Finally, despite the fact that a scheme like P2PC had not, to our knowledge, been formally tested, it sat sensibly within the broader DA context. The scheme was well supported by women's advocacy agencies locally, whilst in the UK, the creation of DA Courts, specialist advocacy services and the establishment of Multi-Agency Risk Assessment Conferences designed to prioritise the safety of victims, stand as complementary developments. Police Scotland's role in providing leadership for this agenda has been described as "exemplary" (Scottish Government, 2014).

We found nothing therefore to suggest dramatic implementation failure. Rather, presenting in terms of low ambiguity/low conflict, we had somewhat reasonable expectations that while the pilot would be relatively straightforward in implementation terms we might uncover discretion in relation to the implementation of risk categories and perspectives of the role of the pilot in contributing to better communication with primary care. Instead, our expectations were confounded by early evidence that the intervention, rather than exhibiting variations in implementation, had failed to materialise. In our attempts to understand why this might have been the case we drew into view two aspects of context 
that were less salient through a street-level lens: the work done by non-human actors; and, the impact of large-scale organisational change.

To relate to the first, the low up-take of P2PC was unmistakably indicative of the scheme's failed introduction, with less than four percent of potential notification letters actually being sent to GPs. Of more marked significance here, however, was the difficulty for the police in calculating this figure. The implementation pathway of P2PC was described by Police Scotland as "untrackable" within their existing data systems. Though data pertaining to this were routinely collected insofar as all letters sent to GPs were to be logged, Police Scotland categorically stated that their databases could not be searched to determine what proportion of eligible victims were visited let alone asked to consent to a GP notification letter under P2PC. Each case had to be hand searched. Through this we began to appreciate the ways in which extant technologies sat within and between the scheme's practices, and came to regard the database as a potential player in mediating its (non)implementation.

As we turned to consider potential explanations for such low level implementation, our attention was drawn to the second mechanism: organisational change. In 2013 , the world of policing in Scotland beyond P2PC changed in ways that dwarfed the intervention. In short, during the time-frame of P2PC's inception and introduction Scotland's police forces were merged, and P2PC was piloted across units designated Legacy Strathclyde. This brought significant organisational upheaval and, in relation to DA policing, new procedures, risk assessment tools, databases and significant staff relocations beyond the normal shifting deployments. More specifically, frontline officers now had to use a different assessment tool, input their own data and utilise a new database. The new database was viewed as a backward step by senior figures in DA support services, who postulated the move was to signal that Strathclyde Police's ways of working shouldn't automatically shape the practice of the new Police Scotland, even though Strathclyde was recognised to be at the forefront of DA policing. This not only added to our sense that technology was an active player in the implementation of P2PC, but, remaining alert to the fact that P2PC took place within a context of extensive organisational change, we reappraised the intervention not as the salient 'new' poised to be embedded into existing practices, but as a rather insignificant 'new' plunged into the even newer. 


\section{Normalisation Process Theory - another way?}

Seeking a frame more suited to understanding blanket implementation failure as well as foregrounding non-human actors and organisational change, we moved to adopt NPT. First developed through health research, NPT explains how new practices make their journey into work routines, and draws attention to both individual and collective action, and the importance of material and social contexts in which such actions unfold. NPT offers to delineate how and why new practices become normalised within professional repertoires. Importantly, it retains a discretionary lens whilst recognising that social factors that "promote or constrain particular expressions of agency ... do not automatically rest on individual cognition and volition and include extant vocabularies and repertoires of interaction, normative frameworks and belief systems, symbolic and material resources, power relations and legitimating authority" (May and Finch, 2009, p. 538). Emphasising the emergent properties of organisations and systems of professional routines which within broader structures operate as the context for new practices; and the work that is done by professionals as they individually and collectively encounter, adopt, and absorb (or resist) new work that is to be inserted into existing daily routines, NPT "emphasizes the fluid, dynamic and interactive processes between context, actors and objects" (McEvoy, et al., 2014 , p. 2). From this, it follows that what is being implemented is not a single task but "an ensemble - of material and cognitive practices" (May, 2013, p.2).

NPT has been applied predominantly within the health care field (see for example: McEvoy et al., 2014; Bamford et al., 2010; Murray et al., 2011; May et al., 2018), including in relation to DA (Hooker et al., 2015). Since its development in 2009, it has become a well-established analytical tool in this domain via its four constructs. 'Coherence' refers to the meaning ascribed individually and collectively to a new set of practices; 'cognitive participation' denotes commitment to engage with the new; 'collective action' addresses how it is that the work does/does not get done; and 'reflexive monitoring' relates to the processes through which practitioners decide whether new approaches are beneficial and lends, ultimately, to the normalisation of new practices.

\section{NPT: for data capture and data analysis}


As set out previously, this paper is concerned with the following research questions:

1. To what extent does NPT help reveal the (non)implementation of P2PC?

2. How does an NPT analysis differ from other approaches for understanding policy implementation?

3. What does this mean for our understandings of policy implementation?

Having recognised $\mathrm{P} 2 \mathrm{PC}$ 's failed introduction early on, we designed our implementation research with NPT to the fore. In elucidating NPT we used the theory's sub-constructs as questions which helped us to unpack NPT in relation to P2PC. This made the value of the framework more obvious, both as we shaped our analytical concerns and subsequently developed our interview schedule. This translation of theory into interview questions is detailed here [online resource]. We deployed this approach in both individual and group settings, with staff from five Legacy Strathclyde DAIUs covered by the pilot (total of 15 participants). These police officers had direct responsibility for investigative policing of DA within each division. Subsequently, we conducted interviews with 20 frontline response and community police officers, purposively selected within each division to give optimum variation in relation to, gender, geographical location and knowledge of the pilot. Across focus groups and interviews we interviewed a total of 35 police officers, with the DACU acting as gatekeeper for participant recruitment. All sessions were audio-recorded and transcribed with the signed, informed consent of participants, and conducted in line with prior approval from the College of Social Science Ethics Committee at the University of Glasgow.

As a research team we held regular data meetings to discuss emerging themes and to resolve coding ambiguities. These meetings also discussed NPT in relation to other bottomup policy implementation approaches with which the team were more familiar, including whether NPT could capture the theme of discretion which we'd previously anticipated as being analytically important and which we coded for deductively. We turn in the next section to our analysis of the data using NPT. Neither gender nor geographical location shaped responses to our questions. Responses received were remarkably similar; we highlight below 
where variations were found. We end by discussing this analysis in relation to policy implementation theory.

\section{An NPT analysis of P2PC}

\section{Coherence}

P2PC appeared to enter police practice as already sensible for DAIU staff. Overwhelmingly evident was a view that multi-agency communication was commonplace. Making referrals was presented as a routine aspect of processing DA incidents, such that sending out notification letters to GPs was described simply as "tweaking what we already do" (DAIU5). $\mathrm{P} 2 \mathrm{PC}$, however, was of course different from other practices in one key way: the requirement for signed consent. This requirement was introduced by a new post-holder. Whilst efforts were made by the DACU to change this to the verbal agreement that satisfied other interagency referrals, signed consent remained. In practice this meant that DAIU officers couldn't process women for the scheme by phone, but had to physically visit women or rely on frontline officers to collect consent for GP referral. As we shall see in the following section, such requests were rarely put in context or with the specific command of obtaining signed consent. This variously resulted in verbal agreement, or even more haphazard examples of victims' signatures scrolled in attending officers' note-books, supposedly signalling consent for all multiagency referral (but not specifically for P2PC).

It is interesting, then, that the thing which to our mind defined P2PC apart from other practices was rarely raised when the participants talked directly about what P2PC was. Indeed, this feature was treated more as a footnote to the scheme, something that could potentially get in the way due to its more resource intensive nature but largely incidental. P2PC in the main was described not for how it stood out from existing practice, but rather, for how it merged in. We will discuss the mode by which P2PC was introduced in the next section, but relevant here is the fact that it was only as research participants that staff discussed P2PC collectively as practitioners. The lack of opportunities for collective understanding and planning prior to the start of P2PC was not lost on DAIU staff, as one participant expressed, "[I]t would have been helpful if we' $d$ all sat round a table before, and if we knew what each other was doing" (DAIU02). 
Likewise, whilst these same practitioners appeared to grasp their roles in enacting P2PC in a hypothetical sense, in reality the low level take-up of the scheme is revealing of the narrow scope for staff to get to know the feel of enacting P2PC in a more meaningful way. It is interesting, then, that staff nonetheless appeared quick to identify benefits of P2PC. The referral letter, for example, was thought key in opening up avenues of support, acting as a catalyst for those who might find it impossible to see beyond their current abusive relationship. P2PC was seen, therefore, as an ice-breaker - "that bridge has been crossed for them" (DAIU03) as one participant noted - and a highly potent one at that:

[GPs have] got a ten second ... snapshot to see that person and not know their history, and you're not always going to be guaranteed to see that same person. So if you see something in their record that's highlighting or flagging something up, if you're a locum that's covering for somebody, then it gives you a wee bit of history that can paint a picture for how you actually deal with what's being presented to you (DAIU05).

Whilst at first glance we might read here a seemingly well considered assessment of the scheme, it must also be noted that such comments relate to P2PC in theory, a notion honed through a much broader experience of multi-agency DA working. As such, this assessment speaks far less to DAIU officers' understanding of P2PC in practice, signalling instead their lack of experience of P2PC specificities. Indeed, also evident in the evaluation was a lack of any collective sense of the purpose of P2PC, the wider implications of which are immediately apparent when we see that some specialist officers were unable to communicate its purpose to victims. As one participant described it: "We've not really known how to really sell it ... I think, if we'd known more about it, we could probably have had loads more referrals" (DAIU02).

Frontline and DAIU officers' experiences were both similar and different. Different insofar as almost all frontline and community officers who we interviewed had no knowledge of the scheme, despite their role in administering P2PC as part of their already existing interagency referral work. Yet in spite of evidence that P2PC visibly entered the frame and was meaningful for DAIU officers at least, the lack of sense-making opportunities for all staff to understand it as a specific, separate and distinct piece of work; to plan for its actual 
administration; and to collectively imagine the concrete values and benefits of P2PC specifically, meant that P2PC was far from coherent. Therefore, NPT helps reveal that in the case of DAIU staff the scheme was fated only to be meaningful in terms of existing practice, rather than an entity in its own right; whilst for frontline officers, P2PC simply remained invisible.

\section{Cognitive Participation}

Data collected across DAIU officers presented a consistent account of P2PC's introduction: units received email communication from the central DACU, informing them that they were to incorporate an offer of GP referral into their daily practice. No training was offered nor protocols provided; no formal documentation existed and staff weren't canvassed for their views. Quite simply P2PC was one day absent within the materials and frames for policing DA incidents and then suddenly present without explanation. Interestingly, this strategy of initiation was not presented to us as unusual. In focus groups and interviews we heard that responding quickly and unquestioningly to directives was considered normal police work. Implementation was not a process but a command, with assumptions of compliance rather than engagement.

This meant that P2PC was communicated on the narrowest need to know basis, and crucially, only staff charged with the initial delivery of the scheme had knowledge of it; the broader cohort of staff members with the potential to be affected by the new routine did not. In practice, this meant that frontline officers - often the first responders who themselves routinely engaged in referrals - were not primed on this particular scheme. This not only created operational difficulties for DAIU officers but meant that when frontline officers did ask women about GP contact within their existing activities of interagency referral, they failed to consistently take the signed consent necessary to invoke the forward movements of P2PC. As one frontline officer put it,

being $100 \%$ honest, when I ask them, 'Do you give us consent?' I don't say separately, 'Are you also giving consent to the GP?' ... I don't think anybody segregates the two (FLO01). 
This clearly signals that a collective figuring out of P2PC in practice was not only lacking between DAIU staff, as we outlined in the previous section, but extended to frontline staff. NPT therefore reveals a crucial step not taken for P2PC: the failure to inform the full range of actors tasked with administering the scheme what to do and why.

Similarly, not only did P2PC's introduction fail to take account of the parallel and entwined work of frontline staff, it also inadequately addressed the resourcing of the scheme within individual DAIU officer workloads. No additional resources were made available for the scheme, the impact of which was immediately apparent from the views of a DAIU officer who confessed: "you're not going to go out and visit someone so they can sign a form, you don't have time" (DAIU01).

Such circumstances resulted in, on the one hand, legitimating DAIU officers' roles in principle (i.e. via the email communication from the central DACU); whilst on the other, acting as a much more tacit, yet powerful de-legitimation of the scheme in reality - as something to be subsumed within existing practice rather than a standalone or outwardly productive scheme. It is no surprise that such modes of initiation and introduction were carried into the ongoing maintenance of the scheme, making P2PC notable only by its absence as time passed.

\section{Collective Action}

Whilst there was little evidence of officers working together to deliver, understand and reinforce P2PC collectively, similarly there were non-human features of P2PC which appear to have worked in tandem with staff to deliver P2PC to such ineffective ends: the means by which DA victims were deemed eligible for the scheme; the signed consent protocol; and the police database system for recording P2PC. In each of these, we found officers exercising discretionary ways of working alongside the scheme's non-human actors, largely in relation to time pressures and administrative workloads. Interestingly, however, officers' discretion not only emerged in response to managing finite resources, but also appeared to press them beyond their existing capacity.

In relation to the first feature, how DA victims' eligibility for P2PC was calculated, DAIU officers talked extensively of how they decided who to visit in person following a DA 
incident. They described over-riding the standardised calculation for identifying high-risk victims, confiding in us the poor predictive accuracy of such measures. Instead, decisions over who was "high-risk" were informed by more nuanced means, including professional experience and communal knowledge about specific individuals in addition to risk assessments from attending officers. The following illustrates this discretion in action as a DAIU officer describes how alarm bells are rung by the seemingly innocuous:

Sometimes you will get a call and it's quite trivial...But obviously something's happened that's made them pick up the phone and phone the police. And you just think ... "That's interesting" ... "There must be more to this (DAIU04).

Despite such desires to engage with a wider cohort of victims, and the variations apparent in actual practice, in reality it appears that limited resources won out.

This brings us to the reason for its initial allocation to DAIU officers, and the second feature outlined, the requirement for signed consent. This particular condition of P2PC meant that it was far more contingent on DAIU resources than if verbal consent had been allowed, in practice making it much more difficult for staff to administer. As such, identifying suitable candidates for the scheme required not simply the identification of a high-risk victim and/or a DAIU officer willing to exercise discretion, but paper, pens, shift patterns and vehicles, for example, were also required. Again, there was some evidence of DAIU officers' discretion in dealing with this aspect of the scheme, but in a much more perfunctory fashion, apparent in the varied manner in which officers sought consent. For some, the act was viewed as a mandatory "bland statement" (DAIU3) to be read out to victims. For others, it was "a judgement call depending on the reaction you get [from victims]" (DAIU02). In a similar way the guiding rationale for the scheme itself was variously attributed by participants, ranging from accepting the potentially significant impacts for women to a rather more cynical and less common view that sending information to GPs was a form of 'arse-covering', as the two quotes juxtaposed below reveal:

The fact that I'm telling your GP might mean that you don't have that initial embarrassment factor having tae tell him 'I'm the victim o' 
DA". I'm doing that for you and you can go in there wi' your head held high because the GP will know it's not your fault'. (FLO02).

Say like a victim had been murdered, and one of the things [the police] could maybe do is look and say 'Right, a GP referral letter got sent on that date...I think [...] with DA... what they do like to do is just cover your arse, I think ... it's just, 'well, we've done that'. So that if anything did happen...we've had that in place (DAIU03).

Third, in addition to such obvious examples of how resources hindered the implementation of $\mathrm{P} 2 \mathrm{PC}$, we also found that discretion manifested in relation to the administrative side of P2PC. While a record of letters sent to GPs were to be logged within the police database, we quickly realised that data were incomplete. As one unit reported:

If you remember to put it on the spreadsheet then good, but sometimes, if you've got a mountain of stuff to get through, it could get missed (DAIU04).

Moreover, even in instances where such data were collected, as discussed earlier, there was no search facility to allow ready calculation of total numbers of letters. Databases needed to be searched per incident to provide this information. Thus, requests for information relating to GP notification letters required additional work on the part of DAIUs. In reality, this meant that the database system charged with recording the practice of the scheme made it impossible to monitor in any meaningful way, in effect rendering incidents invisible after the point of their entry.

Finally, in addition to the (un)productive presence of non-human actors in relation to P2PC, NPT shows the individualised nature of discretionary acts that emerged in relation them. The choice to ignore risk calculations, to ask directly or indirectly for consent, to accept the stated rationale for the scheme, to fail to log letters in the database - these were largely isolated acts, never lending to a collective discussion of working together, nor a visible reinforcing (or indeed, a determined weakening) of P2PC. They occurred but never iterated to become something more; lacking the reinforcing cycle which NPT deems so vital. Further, without significant strategic work to develop and disseminate the purpose and priority of P2PC as a tool within the multi-agency repertoire, the discretionary acts of DAIU officers 
allowed the intervention to hover in sight of practice for some frontline staff without ever bedding in. That is, it is not simply that P2PC couldn't be effectively practiced that led to its obvious (non)implementation, but rather, as NPT reveals, the lack of resources coupled with the lack of sense-making and collective monitoring spaces, meant that nothing even resembling the work could be brought into being let alone sustained.

\section{Reflexive Monitoring}

In view of the evidence of a lack of reflection surrounding the planning and introduction of P2PC that NPT has helped reveal thus far, it is no surprise that monitoring was similarly absent. Numerical data collected - visits made, letters sent, consent refused, for example were not routinely interrogated nor acted upon. Similarly lacking was any qualitative assessment of P2PC. We were told by DAIU staff that the views of key actors were not sought from a strategic perspective. Likewise, when asked about communication between DAIUs, the response shared across units was "we don't really have any contact with other [units]" (FG02). As with the introduction of P2PC, there was little sense from participants that this lack of consultation on progress was unusual. Rather, the expectation to do new things without explicit reflection was considered "normal". As one respondent said:

I think it's just a case of, it's a force procedure and you just need to follow the procedure and do what you can do, when you can do it (DAIU04).

In spite of a lack of official channels for reflection we nonetheless did hear a more informal style of appraisal come forth in judgements made about the pilot. These included reflections about how verbal consent would increase the number of victims being offered the opportunity for a notification letter to be sent and views on the need for better communication flow between police and primary care (specifically GPs). But again, this was only on our prompting; the focus groups were the only opportunities for the principal actors to collectively discuss P2PC.

The lack of official monitoring of P2PC meant that there was nothing to feedback into practice. DAIUs stated that they had neither received (nor sought) feedback from frontline officers or other colleagues involved in obtaining written consent from victims. Likewise, 
there was no indication across units that they received feedback from above. Finally, interviewees discussed the lack of feedback received from those in primary care and also from victims themselves:

No victim's ever come back and said 'oh I went to my doctor's the next time, it was great because they knew about this and I didn't have to go through it' (DAIU02).

For some participants, the lack of confirmation on how information was being used was unproblematic. For others, the overall coherence of the pilot was blurred by an absence of information about its utility. Of particular concern was whether GP referral letters fulfilled their perceived remit of opening up routes to improved support for women. As one participant surmised, "we get absolutely nothing back ... they could be getting filed in the bucket" (DAIU02).

\section{Discussion: Illuminating the (non)implementation of P2PC through NPT - lessons for here and elsewhere}

The story told at the beginning of the paper might have concluded in our conceding to the blanket implementation failure of P2PC: nothing to see, nothing to discuss. Yet as our NPT analysis suggests, there is much to draw out from this case that has relevance for understanding the non-implementation of this particular policy. This in turn, we argue, necessitates the consideration of how we understand implementation more broadly. In this final section of the paper we now turn to discuss these two topics.

\section{NPT for P2PC}

Looking across our analysis, NPT reveals that P2PC was rendered invisible not through single acts or decisions, but in the coming together of a number of factors:

First, NPT reveals that P2PC was given restricted view in terms of who within the police saw it, and made institutionally hard to read by the lack of formal protocols and procedures in its introduction. The lack of space afforded to key actors for the development of their collective and individual roles in P2PC contributed greatly to this, as did the failure to introduce the scheme to frontline staff. 
Second, NPT shows that what eventually manifested was a scheme that was impossible to deliver due to the unexplained shift in staff roles required, and its heavy reliance on absent resources such as DAIU officer's time and transport. We see that all actors - DAIU and frontline staff - had limited opportunity in which to practice P2PC, to make it visible, and to embed it through such activities.

Third, NPT highlights how any opportunities that did arise to shine a light on P2PC were fatally compromised by a failure to instigate systems of monitoring and learning. The ability of Police Scotland's database monitoring systems to make instances of P2PC uncountable and therefore invisible after the point of their entry is one example, as is the lack of space for individual and collective reflection.

Finally, NPT draws our attention not just inward to practice, but outward to context, and we can appreciate the ways in which P2PC was undeniably dwarfed by the massive and rapid organisational shifts towards a unitary policing model in Scotland. What initially presented as fertile ground for P2PC's introduction became much less hospitable in this context. In the redeployment of key actors, and the merging of systems and ways of working, the usual processes through which a intervention might be planned, resourced, introduced, monitored, and eventually bed in and become P2PC, were arguably neither obvious, nor available.

Contradictions exist between these factors. In P2PC's introduction, we witness a command and control culture which simultaneously engenders an unquestioning approach to new directives and willingness to disregard these if they are not followed up by subsequent management communications. In terms of practice, the requirement for signed consent, though easily dismissed as trivial, becomes recognisable as a central factor in the incoherence of the pilot, being both too similar and too different from existing practice. In monitoring and learning, we see an organisation on the one hand willing to commit significant staff resources to our external evaluation, whilst on the other demonstrating a lack of interest in the ongoing internal evaluation of working practices. Looking to context, the massive shifts that occurred around the time of P2PC's roll-out can be made to account for the inhospitable conditions described above, whilst we simultaneously remain aware of 
longstanding features of police work - such as command and control style, lack of evaluation culture (Neyroud and Weisburd, 2014) - that are similarly unsupportive to the embedding of a new intervention.

Under NPT, however, we are able to reappraise these circumstances not as paradoxes as they first appear, but different facets of the intervention. Police officers can both follow commands whilst finding spaces of discretion in which to deviate from their instruction actors are multi-dimensional and their "goal-orientation ... may include resistance, subversion or reinvention, as well as affirmation and compliance" (May and Finch, 2009, p. 544). P2PC can be both too different and too similar - it is not a single entity but an "ensemble of activities" (May and Finch, 2009, p. 540). Police Scotland can commit to external evaluation whilst being inattentive to internal review - these two tasks draw in external and internal actors, and in doing so attend to different parts, different drivers, of the network. Finally, context does have a role to play but does not account for everything just as social processes under an intervention "can be transformed by the proximity of, or interaction with, some other process or event", these same processes are themselves transformed "as they are produced and reproduced" (May and Finch, 2009, p. 548). And it is in this commentary that we see one of the greatest strengths of NPT- we don't need to pick between these scenarios, nor establish causality and hierarchy between them. Our attention is not compelled and fixed within the binary choices of micro or macro, individual or collective, material or social, nor indeed held to top-down or bottom-up sensibilities. Rather, our attention is drawn across all of these frames, and to the interactions between them.

\section{Expanding connections between NPT and broader implementation theory - reflections and concluding comments.}

In the case of P2PC, NPT has offered us the ability to discuss policy implementation where it appeared no policy had been implemented. What does it offer implementation studies more generally? Let us start with a note of caution. In a recent review of NPT studies, McEvoy, et al. noted a distinct danger in donning NPT like a "conceptual straitjacket" (2014, p. 11). At points in our own analysis we encountered the ease at which we might slip into deploying NPT as a tick box exercise, made all the harder to resist given the existence of an NPT toolkit website designed by May and Finch, amongst others ${ }^{3}$. While we did resist it, the toolkit 
website represents a tendency for NPT to be applied unthinkingly. We would argue, however, that there is no need to do and write an NPT analysis in this way (indeed, the recent review by May and colleagues (2018), demonstrates that not all users of NPT do use it in the implied linear fashion). Rather, NPT might be used to add colour to a thematic discussion, it might inspire vignettes of implementation interactions between human and non-human actors, context and discretion. And, should future users turn to NPT with imagination, then it does have a lot to offer.

In terms of NPT's potential value in this regard, this paper began with reference to the traditions of top-down and bottom-up and to the literature on synthesised approaches. The potential value of NPT can and has been understood as joining such debates, leading some to describe it as sitting somewhere between a top-down or bottom-up approach (Fox, et al., 2017). Such a characterisation can certainly be appreciated via the ways in which NPT prompts its user to flit between context and actor, orders and discretion. In doing so NPT facilitates a more holistic understanding of the ways in which the eventual routines of new practices might deviate from policy-on-paper.

Of particular worth in this regard is the ways in which NPT draws attention to the policy work that is done in spaces not contained within the top-down activities of devising policy, and the bottom-up responses in their delivery. How an actor receives and interprets instructions prior to acting is policy making; research, such as ours, which prompts actors to reflect on their practice has the potential to be generative. In directing researchers to individual and collective cognitive acts through the constructs of coherence, cognitive participation, collective action and reflexive monitoring, NPT helps us appreciate the quieter, more tacit, less certain activities that take place to bring sense, meaning and indeed form to policy. NPT's strength here is further complemented in its ability to bring into view a much broader range of categories that matter. From things that sit on the outside of interventions (such as profound organisational change), to things not commonly considered as actors (a police database) - all processes, events and things have productive potential in NPT.

We would argue, however, that NPT's greatest strength does not lie in plugging our knowledge of implementation gaps by adding practices, acts and categories otherwise 
missed, but rather, in the way it prompts us to look to the productive interactions between top, middle and bottom sites of policy making. It is here that we begin to appreciate the many steps of policy implementation that exist between paper and practice; between design and discretion. A simple command (obtain signed consent) can be met with an ill-prepared actor(frontline officers), and in turn produce an mis-actioned request (verbal consent obtained), and then an un-actionable intervention (P2PC disappears). Actors can equate the learned theory of their existing practice (interagency referral) with the imagined benefits of a new scheme (P2PC), and in doing so materially erase the subtleties of its difference (P2PC becomes interagency referral). These individual and collective, cognitive and concrete acts are not errors or missteps, arguably they are the policy (in)action.

Thus we begin to appreciate not simply what NPT might add to implementation research, but how it might also change it. We end this paper with a series of questions. First, in thinking through the interactive and productive acts of implementation that occurred (not just in the elite spaces policy design, and the street-level actions of policy delivery, but also moments of data-entry and (failed)visits to a DA victim's home) we are faced with the consideration of what it is that implementation research is trying to achieve. In this discussion we have praised NPT for its utility in helping to see that which might otherwise remain invisible. Matland (1995), however, was critical of Elmore's work (1979) for stopping at being useful, commenting "as a tool, Elmore's discussion is useful; as a theory, however, it lacks explanatory power" (1995, p.151). This raises an interesting question as to the extent to which it enough for implementation research to be a tool that enables researchers to understand and learn from individual policy cases? Or whether, as per May's own moves towards a "general theory of implementation" (2013) indicate, we must ultimately seek to join up the concepts and arrive at a theory with predictive power?

Second, at the beginning of this paper we discussed concerns that understanding street-level bureaucracy within the tropes of top-down and bottom-up reinforces a normative understanding of the policy process, and misses the extent to which Lipsky's work was concerned with complex organisational behaviour (Brodkin, 2013). NPT has effective reach in both capturing and producing some of the complexity of policy making; how might it fit 
theoretically with street-level sensibilities, and vice versa? And what scope might there be for developing NPT and street-level bureaucracy synergistically?

Third, and related, NPT has sparked our consideration of the extent to which roles played by non-human actors are significant to understanding policy. It should be of note that the field in which NPT was developed, and indeed, where it has had its biggest impact, is in health studies research. Certainly, the Science Technology and Society (STS) and the Actor Network Theory (ANT) traditions from which NPT hails have considerable reach in this field. There is an undeniable need for their efforts to look beyond human actors, given the presence of automation and the highly technical nature of policy responses in their field of study. Policing, and non-health policy contexts more generally, have been traditionally far less reliant on technological support to assist human-actors in delivering policy in the ways that the medical profession, and science more broadly, has done. Arguably this has enabled human discretion to manifest, iterate, and become policy, or at very least, given policy researchers good cause to look to such activities to understand delivery. There is, however, an increasing presence of technological assistance in policing and other domains, and with this comes a need to think carefully how we might understand contemporary policy implementation. How, for example, does the wearing of a camera on a police vest mediate delivery of a DA response? In another domain, might the application for state benefits via an automated telephone call dictate the recipients of support? And what then becomes of the role of human discretion within such activities; the explanatory power of street-level insights (Buffat, 2015); the importance of attending to so-called "screen-level" bureaucracies (Bovens and Zouridis, 2002, p.177); and the value of NPT for helping us rethink our current efforts in this area?

To conclude, this paper has shared the analytical dilemmas of a research team charged with evaluating a policy object that ceased to exist as per its design. Because its virtual demise was swift, we were able to alter our evaluation frame and adopt, unexpectedly, a theoretical tool borrowed from health services research and not previously tested within police work. This proved useful in understanding the particular case in ways that we have set out - in unpicking aspects of context, policy coherence, its sell to the necessary actors and the extent to which it arrived acknowledged as a policy whose implementation would be monitored. 
Going beyond the specific case, however, the significance of this paper rests in its contribution to broader policy implementation debates concerning the precise value and purpose of implementation theory, and the extent to which synchronicities between NPT and street-level bureaucracy theories need to be co-examined, especially in relation to properly understanding the interactions between human and non-human actors as policy and practice moves further in drawing on digital and technological tools in everyday service delivery.

Acknowledgements: This work was supported by the Chief Scientist Office of the Scottish Government under Grant CZH/4/990. 


\section{References}

Bamford, C., Heaven, B., May, C. and Moynihan, P. (2012). Implementing nutrition guidelines for older people in residential care homes: a qualitative study using Normalization Process Theory. Implementation Science, 7:106, pp. 1-13.

Bovens, M. and Zouridis, S. (2002). From Street-Level to System-Level Bureaucracies: How Information and Communication Technology is Transforming Administrative Discretion and Constitutional Control. Public Administration Review, 62:2, pp. 174-184.

Buffat, A. (2015). Street-Level Bureaucracy and E-Government. Public Management Review, 7:1, pp. 149-161.

Brodkin, E. Z. (2012). Reflections on Street-Level Bureaucracy: Past, Present, and Future. Public Administration Review, 72(6), pp. 940-949.

Cairney, P. (2012). Understanding Public Policy: Theories and Issues. Palgrave Macmillan: Basingstoke.

Elmore, R.F. (1979). Backward Mapping: Implementation Research and Policy Decision, Political Science Quarterly, 94, 4, pp. 601-14.

Feder, G., Davies R. A., and Baird, K. (2011). Identification and Referral to Improve Safety (IRIS) of women experiencing domestic violence with a primary care training and support programme: a cluster randomised controlled trial. Lancet, 378, pp. 1788-95.

Fox, C., Grimm, R., Calderia, R. (2017). An Introduction to Evaluation. Sage: London.

Gibb, K. (2015). The multiple policy failures of the UK bedroom tax. International Journal of Housing Policy, 15:2, pp. 148-166.

Home Office (2012). Cross-Government definition of domestic violence: a consultation summary of responses. Home Office: London.

Hooker, L., Small, R., Humphreys, C., Hegarty, K. and Taft, A. (2015). Applying normalization process theory to understand implementation of a family violence screening and care model in maternal and child health nursing practice: a mixed method process evaluation of a randomised controlled trial. Implementation Science, 10:39.

Humphreys, C., Thiara, R. K., Regan, L., Lovett, J., Kennedy, L. and Gibson, A. (2005) A Preliminary Evaluation of the Metropolitan Police Domestic Violence Risk Assessment Model (SPECSS+). University of Warwick: Warwick. 
Hupe, P., Hill M., and Buffat, A. (2015). Understanding Street-level Bureaucracy. Policy Press: Bristol.

King, A., \& Crewe, I. (2013). The blunders of our governments. London: One World.

Kirby, S. (2013). Police effectiveness: Implementation in theory and practice. Basingstoke: Palgrave MacMillan.

Lipsky, M. (1980). Street-Level Bureaucracy: Dilemmas of the individual in public services. New York: Russell Sage Foundation.

Mackenzie, M., Gannon, M., Barton, D., Cosgrove, K., Feder, G., Stanley., N. (2016) Chief Scientist Office Final Report: Police To Primary Care: Police to Primary Care: Testing the feasibility and acceptability of a high risk domestic abuse referral pilot.

Matland, R. E. (1995) Synthesizing the Implementation Literature: The Ambiguity-Conflict Model of Policy Implementation. Journal of Public Administration Research and Theory, 5(2):145-174.

Mazmanian, D. and Sabatier, P. A. (1989) Implementation and Public Policy, University Press of America: Maryland.

McConnell, A. (2010) Policy success, policy failure and grey areas in-between. Journal of Public Policy 30(3), pp. 345-362.

McEvoy, R., Ballini, L., Maltoni, S., O'Donnell, C. A., Mair, F. S. and MacFarlane, A. (2014). A qualitative systematic review of studies using the normalization process theory to research implementation processes. Implementation Science, 9:2.

May, C. and Finch, T. (2009). Normalization Process Theory Implementing, Embedding, and Integrating Practices: An Outline of Normalization Process Theory. Sociology, 43:535.

May, C. (2013). Towards a general theory of implementation. Implementation Science, 8(18): $1-14$

May, CR., Cummings, A., Girling, M., Bracher, M., Mair, FS., May, CM., Murray, E., Myall, M., Rapley, T., Finch, T. (2018) 'Using Normalization Process Theory in feasibility studies and process evaluations of complex healthcare interventions: a systematic review'. Implementation Science, 13(80).

Murray, E., Treweek, S., Pope, C., MacFarlane, A., Ballini, L., Dowrick, C., Finch, T., Kennedy, A., Mair, F., O’Donnell, C. A., Ong, B. N., Rapley, T., Rogers, A. and May, C. (2010). 
Normalisation process theory: a framework for developing, evaluating and implementing complex interventions. BMC Medicine, 8:63.

Neyroud, P. and Weisburd, D. (2014). Transforming the Police Through Science: The Challenge of Ownership. Policing, 8(4):287-293.

Robinson A. L., Myhill A., Wire, J., Roberts J., Tilley N. (2016) Risk-led policing of domestic abuse and the DASH risk model. College of Policing.

Sabatier, P. A. (1986) Top-Down and Bottom-Up Approaches to Implementation Research: A Critical Analysis and Suggested Synthesis. Journal of Public Policy, 6(1):21-48.

The Scottish Government (2014) Equally Safe: Scotland's strategy for preventing and eradicating violence against women and girls. The Scottish Government: Edinburgh

The Scottish Government (2016). High Level Summary of Statistics Trend. Available <http://www.gov.scot/Topics/Statistics/Browse/Crime-Justice/TrendDomesticAbuse>, [accessed: 21 December 2016].

\footnotetext{
${ }^{1}$ Whilst no actual figures on the total number of cases during the audited period were made available, a proxy measure derived from the Recency Frequency Gravity algorithm suggests that we might have anticipated around 2,686 incidents in a 12-month period.

2 There were 58,104 reported incidents in 2015-16 (Scottish Government, 2016). There were two ways in which relevant incidents were reported to the DAIUs. Daily, DAIUs received information concerning all DA incidents occurring over the previous 24 hours - these were risk screened by frontline officers - when the pilot was introduced this was derived from the SPECSS identification tool (Humphreys et al, 2005), a forerunner to the DASH (Domestic Abuse, Stalking and Harassment and Honour Based Violence Risk Identification, Assessment and Management Model (Robinson et al., 2016) now used in England and Wales and the Domestic Abuse Questionnaire used in Scotland. In addition, DAIUs receive monthly lists of perpetrators with a high Recency, Frequency, Gravity (RFG) score - an algorithm calculating risk of future perpetration by known offenders. Victims associated with these perpetrators were deemed to be at high-risk of further DA but the score was recognised by Police Scotland to be an inaccurate assessment of risk as well as one lagging behind real time events. All those in this category were to be contacted by DAIU officers. Where signed consent was provided, a notification letter would then be sent to the named GP.

${ }^{3}$ NPT Toolkit available: http://www.normalizationprocess.org/npt-toolkit/
} 